\title{
Motion artifacts in capacitive ECG measurements : reducing the combined effect of DC voltages and capacitance changes using an injection signal
}

\author{
Citation for published version (APA): \\ Serteyn, A. A. M., Vullings, R., Meftah, M., \& Bergmans, J. W. M. (2015). Motion artifacts in capacitive ECG \\ measurements : reducing the combined effect of $D C$ voltages and capacitance changes using an injection \\ signal. IEEE Transactions on Biomedical Engineering, 62(1), 264-273. \\ https://doi.org/10.1109/TBME.2014.2348178
}

DOI:

10.1109/TBME.2014.2348178

Document status and date:

Published: 01/01/2015

\section{Document Version:}

Publisher's PDF, also known as Version of Record (includes final page, issue and volume numbers)

\section{Please check the document version of this publication:}

- A submitted manuscript is the version of the article upon submission and before peer-review. There can be important differences between the submitted version and the official published version of record. People interested in the research are advised to contact the author for the final version of the publication, or visit the DOI to the publisher's website.

- The final author version and the galley proof are versions of the publication after peer review.

- The final published version features the final layout of the paper including the volume, issue and page numbers.

Link to publication

\footnotetext{
General rights

- You may freely distribute the URL identifying the publication in the public portal. follow below link for the End User Agreement:

www.tue.nl/taverne

\section{Take down policy}

If you believe that this document breaches copyright please contact us at:

openaccess@tue.nl

providing details and we will investigate your claim.
}

Copyright and moral rights for the publications made accessible in the public portal are retained by the authors and/or other copyright owners and it is a condition of accessing publications that users recognise and abide by the legal requirements associated with these rights.

- Users may download and print one copy of any publication from the public portal for the purpose of private study or research.

- You may not further distribute the material or use it for any profit-making activity or commercial gain

If the publication is distributed under the terms of Article 25fa of the Dutch Copyright Act, indicated by the "Taverne" license above, please 


\title{
Motion Artifacts in Capacitive ECG Measurements: Reducing the Combined Effect of DC Voltages and Capacitance Changes Using an Injection Signal
}

\author{
A. Serteyn*, R. Vullings, M. Meftah, and J. W. M. Bergmans, Senior Member, IEEE
}

\begin{abstract}
Capacitive electrodes are a promising alternative to the conventional adhesive electrodes for ECG measurements. They provide more comfort to the patient when integrated in everyday objects (e.g., beds or seats) for long-term monitoring. However, the application of capacitive sensors is limited by their high sensitivity to motion artifacts. For example, motion at the body-electrode interface causes variations of the coupling capacitance which, in the presence of a dc voltage across the coupling capacitor, create strong artifacts in the measurements. The origin, relevance, and reduction of this specific and important type of artifacts are studied here. An injection signal is exploited to track the variations of the coupling capacitance in real time. This information is then used by an identification scheme to estimate the artifacts and subtract them from the measurements. The method was evaluated in simulations, lab environments, and in a real-life recording on an adult's chest. For the type of artifact under study, a strong artifact reduction ranging from $40 \mathrm{~dB}$ for simulated data to $9 \mathrm{~dB}$ for a given reallife recording was achieved. The proposed method is automated, does not require any knowledge about the measurement system parameters, and provides an online estimate for the dc voltage across the coupling capacitor.
\end{abstract}

Index Terms-Capacitive electrocardiography (ECG), injection signal, insulated electrodes, modeling, motion artifacts.

\section{INTRODUCTION}

I NTRODUCED by Lopez and Richardson in 1967 [1] and further developed in the last decade, capacitive sensors allow biopotential recordings through insulating materials, e.g., clothing. Since no direct skin contact is needed, capacitive sensors can be integrated in everyday objects, such as beds for longterm unobtrusive monitoring [2] or cars for assisted driving [3]. Recently, capacitive sensors were evaluated for electrocardiographic (ECG) recordings in clinical settings [4], [5].

In the recording of biopotentials with capacitive sensors, interferences due to environmental electrical fields and motion artifacts are problematic and need to be addressed before capacitive sensors can be used in clinical routine. In this paper, we describe our solution to reduce one major type of artifacts:

Manuscript received July 22, 2013; revised June 3, 2014; accepted July 19 , 2014. Date of publication August 15, 2014; date of current version December 18,2014 . This work was supported by Agentshap-NL. Asterisk indicates corresponding author.

*A. Serteyn is with the Department of Electrical Engineering, Eindhoven University of Technology, 5612 AZ Eindhoven, The Netherlands (e-mail: a.a.m.serteyn@tue.nl).

R. Vullings and J. W. M. Bergmans are with the Faculty of Electrical Engineering, Eindhoven University of Technology, 5612 AZ Eindhoven, The Netherlands (e-mail: r.vullings@tue.nl; j.w.m.bergmans@tue.nl).

M. Meftah is with the Patient Care Solutions Group, Philips Research, 5656 AE Eindhoven, The Netherlands (e-mail: mohammed.meftah@philips.com).

Digital Object Identifier 10.1109/TBME.2014.2348178

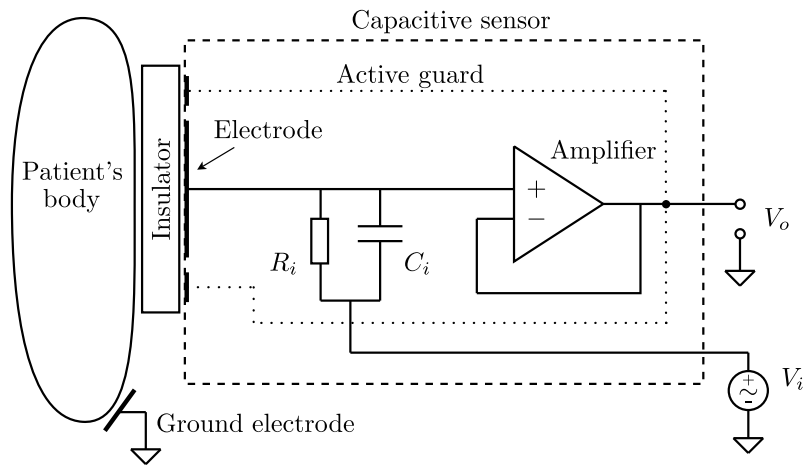

Fig. 1. Capacitive biopotential measurement system composed of a patient's body and a capacitive sensor. The electrode is capacitively coupled to the patient via an insulator, e.g., an air gap. An active guard protects the high-impedance sensor from environmental electromagnetic interferences. A known voltage $V_{i}$ is applied from the sensor ground to track the variations of the coupling capacitance.

the one due to the combination of body-electrode impedance variations and a dc voltage across the body-electrode interface.

\section{BACKGROUND}

\section{A. Capacitive Measurement System}

The design of electronics for capacitive sensors has been studied for more than 45 years but remains challenging [6]. To record biopotentials, an electrode is coupled to the patient's body via a capacitance on the order of a few picofarads and connected to a very high-input-impedance amplifier $(>1 \mathrm{~T} \Omega)$. The electrode and the amplifier together define a capacitive sensor. The coupling of the capacitive sensor to the body defines a capacitive measurement system, as illustrated in Fig. 1 .

In order to limit interferences and allow a reliable and robust measurement, different hardware designs have been proposed in the literature. For example, active guarding and neutralization are commonly implemented to keep the bias resistance $R_{i}$ very high and the input capacitance $C_{i}$ as low as possible [7], [8]. A driven-right-leg circuit (DRL) can also be used to reduce common-mode interferences [7]. In this paper, we consider the basic form of capacitive sensor, without neutralization or DRL, but with an injection signal emitted from the sensor ground terminal as in Fig. 1.

The coupling capacitance depends on the dielectric property of the material at the body-electrode interface. This material can range from an air gap to a thin cotton cloth that can be more or 
less compressed depending on the body-electrode distance. The body-electrode interface can also be a combination of different materials, e.g., a waterproof mattress cover and a cotton bed sheet. In this study, we assume the body-electrode interface to be an air gap to avoid triboelectric effects [9] and be able to focus on one source of artifacts. However, a real-life experiment with a cotton layer is also performed to highlight the challenges related to more complex body-electrode interfaces.

\section{B. Sources of Artifacts}

Three main sources of artifacts for capacitive ECG measurements were identified in the literature: the environmental electromagnetic interferences associated with a poor common-mode rejection ratio of the system [3], [9], the local triboelectric effect at the body-electrode interface [9], and the variation of the coupling capacitor under dc voltage [10], [11]. All three types of artifacts can dominate the others, depending on the measurement conditions (patient grounding, amount of body motion, type of materials at the body-electrode interface, etc.). This paper aims to study and reduce the third type of artifacts.

The dc voltage across the coupling capacitor has different origins. It comes in part from the dc bias current of the amplifier, from electrodermal dc voltages (skin potential), and from the accumulation of static charges (e.g., by the triboelectric effect) on the body surface and in the capacitive sensor. In the presence of this dc voltage, any variation of the capacitance due to motion creates artifacts in the measured signal. We focus thus on artifacts coming from capacitance variations of a charged capacitor. These artifacts are sometimes referred to as microphonics in the literature. Since the dc voltage is mainly created by triboelectric effects, microphonics are sometimes confused with triboelectric artifacts. Microphonics consist in a charge flow due to vibration, while the triboelectric effect consist in a charge generation due to friction. These two effects are often combined: A static charge is generated (creating a transient artifact in the signal) and then modulated by the motion (creating an artifact correlated with the motion) until it discharges or adds to a newly generated charge.

\section{Prior Work}

A theoretical model to study the artifacts generated by variations of the charged coupling capacitor was proposed by Ottenbacher and Heuer in [12]. To undo the artifacts, they solved the inverse system equation. Their method showed promising results in simulation [12] and controlled bench experiments [13]. However, exact knowledge of all the model parameters (system components and dc voltage across the coupling capacitor) is required. This knowledge is often not available, limiting the applicability of this approach for artifact reduction (AR).

Another method for reducing the artifact due to a varying and charged coupling capacitor was recently tested by Eilebrecht et al. in [14]. They used 3-D acceleration signals and combined them to estimate the artifact via a classic adaptive filtering scheme. Their method has the limitation that it requires an additional sensor (an accelerometer) that must be synchronized with the capacitive sensor. Besides, whether or not the accelerometer signal can lead to a good estimation of the artifact has not been studied. Their method is also not automated since the combination of the accelerometer signals that leads to the best rejection of artifacts is selected manually.

As an alternative to accelerometer signals, other signals that correlate with the motion at the body-electrode interface and could potentially be used for artifacts reduction were mentioned, but not exploited, in the literature. A first suggestion was to use an auxiliary capacitive sensor to track changes in the coupling impedance [15]. A second suggestion was to use an injection signal for continuous impedance measurement [5], [16]. This technique is already used in conventional ECG measurement systems with gel or dry electrodes, e.g., in [17]. However, it has only recently been proposed for ECG measurement systems with capacitively coupled electrodes. In these recent investigations, an injection signal is used for channel selection [18], respiration observation [19], artifact localization [20], or impedance measurement in static conditions [21], but not for AR or ECG reconstruction. The first time an injection signal was actually tested for AR in capacitive measurements was in our preliminary work in [22]. The adaptive filter that was used did not exploit any knowledge about the system model. The method showed promising results; however, the optimal learning rate and length of the adaptive filter were obtained heuristically.

\section{PRoposed Method}

We propose to use a time-invariant filter approximation, an injection signal, and a parameter optimization scheme to estimate and reduce motion artifacts caused by variations of the charged coupling capacitor. In addition to AR, our method provides an indication of the variations of the coupling capacitance as well as an estimate of the dc voltage across the coupling capacitor. The method is fully automated and does not require any knowledge about the system component values. The method is designed to perform AR on a single-sensor basis. Hence, no sensor array or auxiliary sensor is needed.

The paper starts with an analysis of the motion artifact based on a model of the capacitive measurement system in Section IV. The main components of the motion artifact are further studied in Sections V and VI in terms of their impact on the ECG measurement in the best- and worst-case scenarios. Section VII describes our method to estimate and reduce the dominant component of the motion artifact. First, a simplified model for the motion artifact is derived from the system model using a time-invariant filter approximation (see Section VII-A). Then, an injection signal and a sliding-window optimization are used to estimate the parameters of the artifact model (see Sections VII-B and VII-C). Finally, the estimated artifact is subtracted from the ECG measurement. In Section VIII, we then consider a by-product of our AR method: the dc voltage estimate. To conclude, the results of the AR in simulation, in a lab bench setup as well as in a real-life scenario are presented and discussed in Section IX.

\section{MOdEL OF THE CAPACITIVE SySTEM}

A capacitive sensor measures the displacement current caused by electrical fields on the body surface. The body surface and 


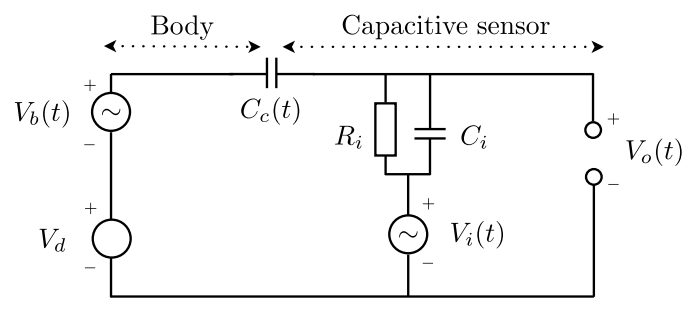

Fig. 2. Equivalent circuit of a capacitive measurement system, adapted from [12]. The dc voltage $V_{d}$ and the time variations of the coupling capacitance $C_{c}(t)$ cause an artifact in $V_{o}(t)$. A high-frequent signal $V_{i}(t)$ is injected to track the time variations of $C_{c}(t)$.

the electrode are insulated from each other and act as the two plates of a capacitor. In these conditions, no galvanic contact between the electrode and the body is required, and the bodyelectrode interface is modeled by a simple capacitor. The capacitance $C_{c}(t)$ of this capacitor is inversely proportional to the distance between the capacitor plates and proportional to the area of the plates. Accordingly, $C_{c}$ varies over time with any motion that occurs at the body-electrode interface.

The equivalent circuit of a biopotential sensor that is capacitively coupled to a body is shown in Fig. 2. This circuit was first proposed in [12]. We adapted it here to include a voltage source $V_{i}(t)$ that represents a known high-frequent sinusoidal signal injected from the sensor ground and used to gauge the time variations of $C_{c}(t)$. The high input impedance of the amplifier in the capacitive sensor, together with the bias resistance of the amplifier and some parasitic capacitances (from pads, traces, etc.), is modeled by a resistance $R_{i}$ in parallel to a capacitance $C_{i}$, both assumed to be time-invariant. The voltage $V_{b}(t)$ stands for the biopotential to be measured, e.g., an electrocardiogram. The voltage $V_{d}$ represents the dc voltage across $C_{c}(t)$. Finally, $V_{o}(t)$ is the voltage at the output of the measurement system. Note that this model assumes a galvanic (resistive) grounding of the patient body.

The time-domain behavior of the capacitive measurement system can be mathematically described by

$$
\begin{aligned}
\left(C_{i}+C_{c}\right) \frac{d V_{o}}{d t}+ & \left(\frac{1}{R_{i}}+\frac{d C_{c}}{d t}\right) V_{o} \\
& =\frac{V_{i}}{R_{i}}+C_{i} \frac{d V_{i}}{d t}+\frac{d\left(C_{c} V_{b}\right)}{d t}+\frac{d\left(C_{c} V_{d}\right)}{d t}
\end{aligned}
$$

where $C_{c}, V_{o}, V_{i}$, and $V_{b}$ are time varying. This equation was, on the one hand, implemented in Simulink (The MathWorks, Inc.) using the parameters of Table I and, on the other hand, solved analytically using standard techniques for differential equations with time-varying coefficients [23]. The analytical solution aims to gain insight into the system behavior, allowing a system approximation as well as the design of a dedicated method for AR. The implementation in Simulink is used to generate data to test our AR method.

In the simplifying case, where $V_{i}=0$ and with the initial condition $t_{0} \rightarrow-\infty$, we found analytically that [22]

$$
V_{o}(t)=s(t)+a(t)
$$

TABLE I

Model Parameters Used for the SimUlation

\begin{tabular}{lccc}
\hline \hline Parameter & Symbol & Value & Frequency band \\
\hline Total input resistance & $R_{i}$ & $\sim 50 \mathrm{G} \Omega$ & 0 \\
Total input capacitance & $C_{i}$ & $\sim 3 \mathrm{pF}$ & 0 \\
Coupling capacitance & $C_{c}$ & {$[0.58] \mathrm{pF}$} & {$[020] \mathrm{Hz}$} \\
DC voltage across $C_{c}$ & $V_{d}$ & {$[-11] \mathrm{V}$} & {$[00.5] \mathrm{Hz}$} \\
Injection signal & $V_{i}$ & $100 \mathrm{mV}_{\mathrm{pp}}$ & $1 \mathrm{kHz}$ \\
Biopotential (ECG) & $V_{b}$ & $1.3 \mathrm{mV}_{\mathrm{p} \mathrm{p}}$ & {$[0.540] \mathrm{Hz}$} \\
\hline \hline
\end{tabular}

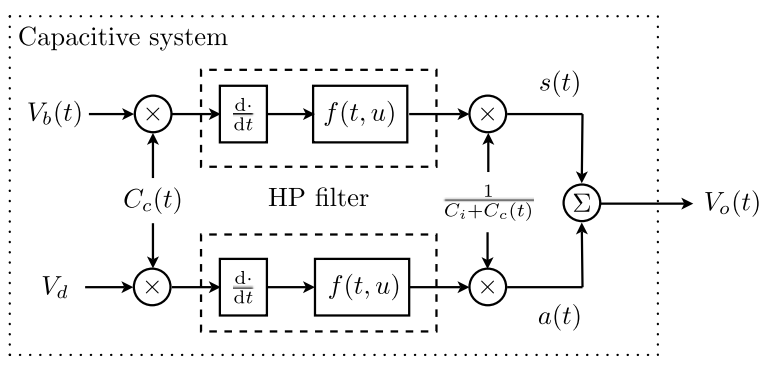

Fig. 3. Model of a capacitive measurement system. The output signal $V_{o}(t)$ is the sum of two terms: $s(t)$, a distorted version of the biopotential of interest $V_{b}$, and $a(t)$, an additive artifact due to the time variations of $C_{c}(t)$ in the presence of a dc voltage $V_{d}$. The differentiator $\frac{d}{d t}$ and the dynamic low-pass filter of impulse response $f(t, u)$ form together an HP filter.

where

$$
\begin{aligned}
& s(t)=\frac{1}{C_{i}+C_{c}(t)} \int_{-\infty}^{t} \frac{d\left(C_{c} V_{b}\right)}{d u} f(t, u) d u \\
& a(t)=\frac{V_{d}}{C_{i}+C_{c}(t)} \int_{-\infty}^{t} \frac{d C_{c}}{d u} f(t, u) d u
\end{aligned}
$$

with

$$
f(t, u)=\mathrm{e}^{-\int_{u}^{t} k(s) d s}
$$

and

$$
k(t)=\frac{1}{R_{i}\left(C_{i}+C_{c}(t)\right)} .
$$

According to (2), the signal of interest $V_{b}(t)$ is dynamically highpass (HP) filtered by the system, providing a distorted version $s(t)$ of $V_{b}(t)$. According to (1) and (3), the output $V_{o}(t)$ further contains an unwanted additive artifact $a(t)$, proportional to $V_{d}$. This model of $V_{o}(t)$ is schematically represented in Fig. 3.

The model parameters $R_{i}, C_{i}$, and $C_{c}(t)$ represent physical electrical components. Their typical values are listed in Table I. The total input impedance of the amplifier $\left(R_{i} \| C_{i}\right)$ depends on the circuit construction and is given here for our measurement system. The lower value of $C_{c}(0.5 \mathrm{pF})$ corresponds to a distance of $3.5 \mathrm{~mm}$ between the body and electrode surfaces, for an electrode area of $2 \mathrm{~cm}^{2}$ and an air gap as insulating layer. We assume that above $3.5 \mathrm{~mm}$ (i.e., below $0.5 \mathrm{pF}$ ), the sensor is not coupled to the body. As for the upper bound, $8 \mathrm{pF}$ corresponds to a cotton layer $\left(\epsilon_{r}=1.3\right)$ of 0.3 -mm thickness as insulating layer. Using an impedance-analyzer (Agilent 4294A), we measured a coupling capacitance of $4.2 \mathrm{pF}$ for one layer of polyurethane and two layers of cotton as body-electrode interface, which further 
justifies the chosen $C_{c}$ values. The frequency content of $C_{c}(t)$ ranges from 0 to $20 \mathrm{~Hz}$. This frequency band was chosen in order to cover motion artifacts due to breathing $(0.1-2 \mathrm{~Hz})$ and body movement $(0-10 \mathrm{~Hz})$, but also due to ballistocardiographic vibrations $(0-20 \mathrm{~Hz})$ [19] and, to a certain extent, artifacts due to transient movement, e.g., a step load of the sensor. The voltage $V_{d}$ across $C_{c}$ is assumed to be constant (dc). However, the impact of a drift or slow variations of this voltage up to $0.5 \mathrm{~Hz}$ will be discussed in Section VIII, which explains the [0 0.5] $\mathrm{Hz}$ bandwidth in Table I. The signal $V_{i}$ has an amplitude of $100 \mathrm{mV}_{\mathrm{pp}}$ to ensure a patient leakage current always smaller than $50 \mu \mathrm{A}$. The ECG bandwidth was limited to $0.5-40 \mathrm{~Hz}$, which is the typical bandwidth of patient monitors in the socalled Monitoring Mode. Choosing this bandwidth also allows us to avoid 50- and $60-\mathrm{Hz}$ interferences and be able to better isolate the one type of artifact under study. Taking a larger bandwidth for $V_{b}(t)$ will have no impact on our AR method as soon as the common-mode interferences such as 50 and $60 \mathrm{~Hz}$ are cancelled beforehand, e.g., using a notch filter or a DRL circuit.

\section{Distortions of THE Biopotential}

Equation (1) shows that the output signal $V_{o}(t)$ has two components. This section focuses on the first component: the distorted signal $s(t)$, as described in (2). Distortions not only consist of an amplitude modulation of the signal of interest $V_{b}(t)$ but also of a dynamic filtering of $V_{b}(t)$. Within the range of values and frequency bands of the model parameters of Table I, several scenarios can be analyzed and simulated.

\section{A. Best-Case Scenario}

The best-case scenario, that is when there is the least amount of distortions, occurs when the coupling capacitance is constant, i.e., there is no motion at the body-electrode interface. In this case, (1) becomes

$$
V_{o}(t)=s_{0}(t)
$$

where

$$
s_{0}(t)=\frac{C_{c}}{C_{i}+C_{c}} \int_{-\infty}^{t} \frac{d V_{b}}{d u} e^{-k(t-u)} d u .
$$

The measured signal in the absence of motion $s_{0}(t)$ is thus a first-order HP version of $V_{b}$ scaled by the time-invariant factor $\frac{C_{c}}{C_{i}+C_{c}}$. In the absence of motion, the sensor may thus be described by a linear time invariant (LTI) HP filter with frequency response

$$
H_{0}(j \omega)=\frac{C_{c}}{C_{i}+C_{c}} \frac{j \omega}{j \omega+k_{0}}
$$

where $k_{0}=\frac{1}{R_{i}\left(C_{i}+C_{c}\right)}$ is the angular cutoff frequency. To ensure that the cutoff frequency of the HP filter remains below the frequency band of $V_{b}(t)$, specific designs of the bias circuit were studied in the literature to make $R_{i}$ sufficiently high [6], [7], [24]. With the parameter values of Table I, the cutoff frequency of our system varies between 0.3 and $0.9 \mathrm{~Hz}$. The sensor may thus filter out some of the very low-frequency components

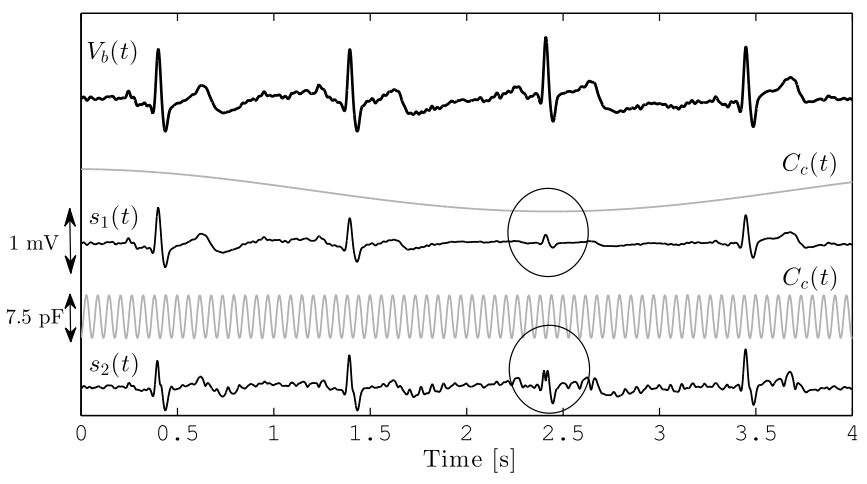

Fig. 4. Two examples of ECG distortions. When the coupling capacitance $C_{c}(t)$ varies at a frequency of $0.2 \mathrm{~Hz}$ between its extreme values, which could represent breathing motion, we obtain the distorted signal $s_{1}(t)$. When $C_{c}(t)$ varies at a frequency of $17 \mathrm{~Hz}$, which is one of the dominant frequencies in the ECG, we obtain $s_{2}(t)$.

of the measured ECG. However, this will not have an impact on the detection of the R-peaks needed to determine the heart rate since most of their spectral energy is contained in the 10 $30 \mathrm{~Hz}$ range [25]. Note that the impact of $C_{c}$ on the measured signal amplitude (scaling effect) is minimal when the scaling factor $\frac{C_{c}}{C_{i}+C_{c}}$ in (6) is close to 1 . Efforts have thus been made in the literature toward reducing the input capacitance $C_{i}$, e.g., by implementing a neutralization circuit [7].

\section{B. Worst-Case Scenario}

In the worst-case scenario, motion at the body-electrode interface makes $C_{c}(t)$ vary between its minimal and maximal values, further distorting the biopotential measurement. Two examples of worst-case scenarios, for a $0.2-$ and $17-\mathrm{Hz}$ motion, are illustrated in Fig. 4. In both cases, the third R-peak experiences the worst-case distortions. The other R-peaks are displayed for comparison purpose. The signal $s_{1}(t)$ in Fig. 4 shows that the signal attenuation is the strongest when the value of $C_{c}(t)$ is the smallest, that is when there is no proper capacitive coupling at the body-electrode interface. The signal $s_{2}(t)$ shows that a high-frequency distance change at the body-electrode interface occurring exactly at the same time as an R-peak of the ECG signal can alter the R-peak shape, however not entirely mask it. Therefore, given the parameters of Table I, the distortions are not expected to prevent a proper R-peak detection, even in the worst-case scenario.

\section{AdDitive ARTIFACT IN THE BIOPOTENTIAL MEASUREMENT}

This section focuses on the second component of $V_{o}(t)$ : the additive artifact $a(t)$. This artifact, as described in (3), is directly proportional to the dc voltage $V_{d}$ and is present only when motion occurs at the body-electrode interface.

\section{A. Best-Case Scenario}

The best-case scenario is encountered when there is no dc voltage $\left(V_{d}=0\right)$ or when there is no motion $\left(C_{c}\right.$ is constant over 


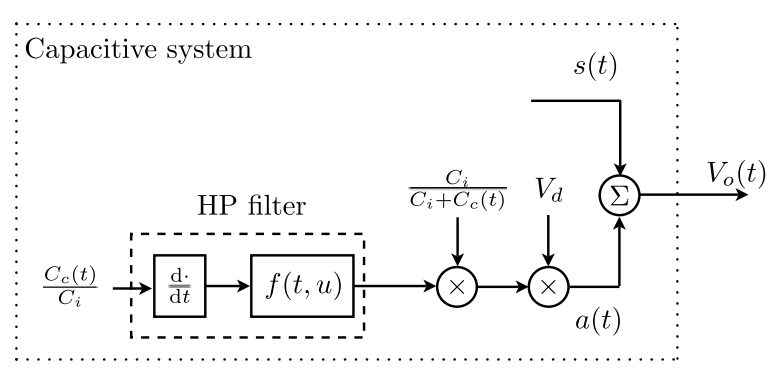

Fig. 5. Model of the capacitive measurement system, adapted from Fig. 3 and with a focus on the additive artifact $a(t)$.

time). In these two cases, the additive artifact is not present:

$$
V_{o}(t)=s(t)
$$

and the measurement is affected by the distortions only.

\section{B. Worst-Case Scenario}

In practice, the dc voltage $V_{d}$ across $C_{c}(t)$ can be hundreds of millivolts or even several volts, depending mainly on the amount of static charge on the body surface or on the textile layers at the body-electrode interface. Since the artifact $a(t)$ is directly proportional to $V_{d}$, in the worst-case scenario, $a(t)$ will completely mask the measured biopotential (see Fig. 9 for an illustration) and may even lead to clipping of the system. Suppressing the additive artifact is therefore a more urgent problem than correcting for the signal distortions.

The impact of $a(t)$ on the biopotential measurement was simulated and evaluated with the amplitude of $a(t)$ as quality metric. We find that a $V_{d}$ value as small as $0.6 \mathrm{mV}$ is already critical. Indeed, for $V_{d}=0.6 \mathrm{mV}$ and $C_{c}$ varying between its extreme values, $a(t)$ has an amplitude of $0.7 \mathrm{mV}$ peak-to-peak, which is similar to the one of the R-peaks when measured on the chest of an adult. In this case, any peak in the motion artifact could be misinterpreted as an R-peak of the ECG and could introduce errors in the R-peak detection and related heart rate computation.

Reducing the additive artifact due to the dc voltage $V_{d}$ in combination with motion is the focus of this paper. The goal is to recover key features of the ECG signal, i.e., the R-peaks, in order to allow an accurate instantaneous heart rate computation or R$\mathrm{R}$-interval time series extraction. When the addictive artifact is entirely removed, the R-peaks become visible again, although the signal distortions remain.

\section{AdDITIVE ARTIFACT ESTIMATION AND SUbTRACTION}

To reduce the additive artifact $a(t)$, we propose to first estimate it as accurately as possible and then subtract it from $V_{o}(t)$. To estimate $a(t)$, we use our model of the capacitive measurement system. As previously shown in Fig. 3 and in (3), $a(t)$ is created from an input signal $V_{d} C_{c}(t)$ that is dynamically HP filtered and then scaled by $\frac{1}{C_{i}+C_{c}(t)}$. By rearranging this succession of events, we obtain the model for $a(t)$ given in Fig. 5. Since, in practice, the dynamic HP filter as well as the model parameters are not known, we propose to approximate

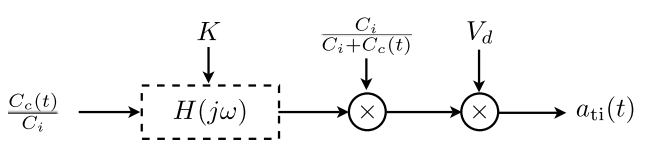

Fig. 6. Approximation of the additive artifact. Step 1: the model of the artifact is simplified: the dynamic HP filter is approximated by an LTI HP filter.

and estimate them based on the injection signal $V_{i}(t)$. The three steps leading to an estimate of $a(t)$ are detailed hereafter.

\section{A. Step 1: Simplification of the Artifact Model Via an LTI Approximation of the Dynamic Filter}

We first approximate the time-varying HP filter of Fig. 5 by an LTI HP filter with impulse response $h(t)$, transfer function $H(j \omega)=\frac{j \omega}{K+j \omega}$, and angular cutoff frequency $K$. In these conditions, the artifact $a(t)$ can be approximated by

$$
a(t) \cong a_{\mathrm{ti}}(t)=\frac{V_{d}}{C_{i}+C_{c}(t)}\left(C_{c}(t) * h(t)\right)
$$

where $*$ indicates the convolution operation. This expression is schematically illustrated in Fig. 6.

\section{B. Step 2: Estimation of the Time-Varying Model Parameters via a Demodulation of the Injection Signal}

The time-varying model parameter $\frac{C_{c}(t)}{C_{i}}$ is not a priori known. To be able to track it, we inject a known sinusoidal signal $V_{i}(t)$ in the system. The voltage $V_{i}(t)$, when applied from the sensor ground as in Figs. 1 and 2, is transferred to the output of the capacitive measurement system according to

$$
\frac{V_{o}(j \omega)}{V_{i}(j \omega)}=\frac{1+C_{i} R_{i} j \omega}{1+\left(C_{c}+C_{i}\right) R_{i} j \omega} .
$$

At sufficiently high frequencies (large $\omega$ ), this transfer function reduces to a real-valued gain

$$
\frac{V_{o}(t)}{V_{i}(t)}=g(t)=\frac{C_{i}}{C_{i}+C_{c}(t)} .
$$

In this case, any variation of $C_{c}(t)$ will instantaneously affect the contribution of $V_{i}(t)$ in the output signal $V_{o}(t)$ via the gain $g(t)$. We measure $g(t)$ directly by demodulating $V_{o}(t)$ at the frequency of $V_{i}(t)$. A frequency of $1 \mathrm{kHz}$ for $V_{i}(t)$ is chosen because, at $1 \mathrm{kHz}$, the imaginary part of $\frac{V_{o}}{V_{i}}$ is less than $0.1 \%$ of its real part (phase shift below $0.3^{\circ}$ ), so the assumption that the transfer function $\frac{V_{o}}{V_{i}}$ is real holds.

The time-varying gain $g(t)$ as defined in (7) represents one of the two time-varying parameters in the model of Fig. 6 . The other time-varying parameter is obtained from $g(t)$ according to

$$
\frac{C_{c}(t)}{C_{i}}=\frac{1-g(t)}{g(t)} .
$$

With the use of the injection signal, the scheme for the artifact approximation given in Fig. 6 is adapted as in Fig. 7. 


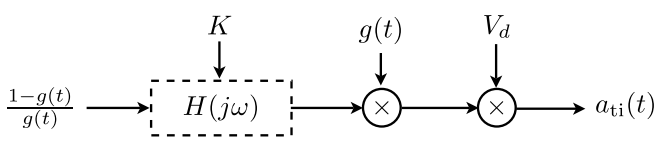

Fig. 7. Approximation of the additive artifact. Step 2: the time-varying parameters of the model are estimated, as in (7) and (8), using the amplitude modulation $g(t)$ of the $1-\mathrm{kHz}$ injection signal $V_{i}(t)$.

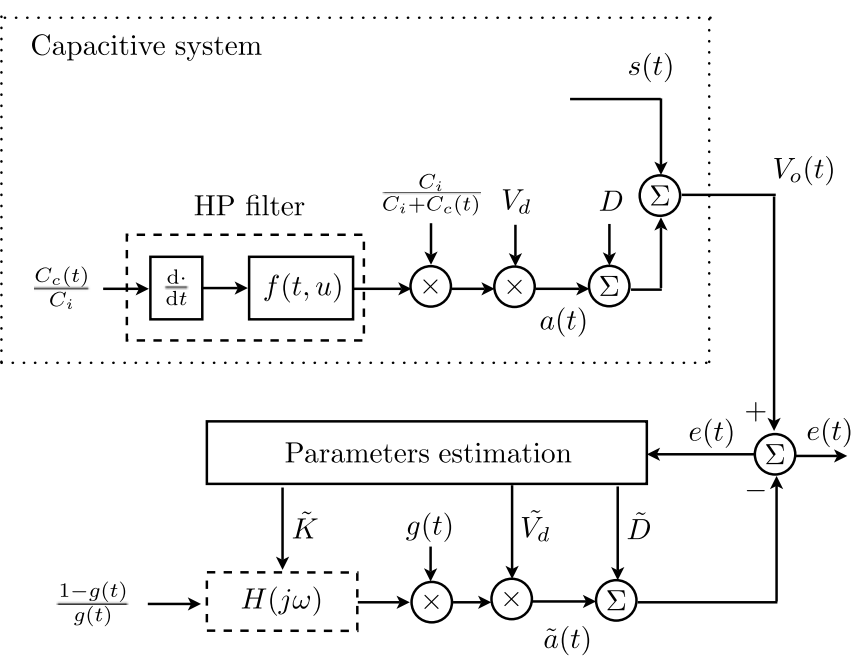

Fig. 8. Identification scheme for the reduction of the additive artifact. An estimate $\tilde{a}(t)+\tilde{D}$ for $a(t)+D$ is provided and then subtracted from $V_{o}(t)$. The computation of $\tilde{a}(t)$ is based on an LTI approximation, an injection signal, and a block least-squares parameters estimation.

\section{Step 3: Estimation of the Constant Model Parameters via a Block Least-Squares Minimization}

The constant (or slowly varying) model parameter $V_{d}$ as well as the time-invariant cutoff frequency $K$ of the filter $H(j \omega)$ remain to be estimated. In practice, an offset $D$ is also present in $V_{o}(t)$ due to offsets of the operational amplifier and must also be estimated.

We want to estimate the scaling factor $V_{d}$, the parameter $K$, and the offset $D$ such that they provide the least-squares estimate $\tilde{a}(t)+\tilde{D}$ of $a(t)+D$. If the motion signal $\frac{C_{c}(t)}{C_{i}}$ is uncorrelated with the signal of interest $s(t)$, the least-squares estimate is obtained when the power of the error $e(t)=s(t)+$ $a(t)+D-\tilde{a}(t)-\tilde{D}$ is minimal. The process is represented by the parameters estimation box in Fig. 8 .

The parameters estimation is performed by a block leastsquares minimization. Since $e(t)$ depends linearly on $\tilde{V}_{d}$ and $\tilde{D}$, the optimum values of $\tilde{V}_{d}$ and $\tilde{D}$ can be computed directly by setting the partial derivatives of the power of $e(t)$ to zero and solving the system of two equations with two unknowns. Regarding the parameter $K$, all values ranging from $1.8 \mathrm{rad}$ [for $C_{c}=8 \mathrm{pF}$ in (5)] to $6 \mathrm{rad}$ (for $C_{c}=0.5 \mathrm{pF}$ ) are tested. For each different value of $\tilde{K}$, the parameter estimates $\tilde{V}_{d}$ and $\tilde{D}$ are recomputed. The set of parameters minimizing the power of $e(t)$ is selected. Since the power of $e(t)$ as a function of $K$ is observed to be convex, a simple gradient descent algorithm can be used instead of scanning through all $K$ values to make the optimization more efficient. The choice of the block length is discussed in Section VIII-B.

\section{VIII. $V_{d}$ ESTIMATE}

Our AR scheme provides, as by-product, an estimate for the dc voltage $V_{d}$.

\section{A. Time Variations of $V_{d}$}

The dc voltage $V_{d}$ models, among other things, the static charge across the coupling capacitor $C_{c}$. In real measurements, this voltage may, on the one hand, drift over time due to a slow discharge of the static charge on the body and, on the other hand, vary in an unknown manner with motion or friction at the bodyelectrode interface because of the triboelectric effect. From the equivalent circuit in Fig. 2, we see that $V_{d}$ and the ECG signal $V_{b}(t)$ cannot be distinguished as soon as their frequency band overlap. The voltage $V_{d}$ can thus be allowed to vary up to $0.5 \mathrm{~Hz}$ in case we are interested in the full ECG shape $\left(V_{b} \in[0.540]\right.$ $\mathrm{Hz}$ ) and up to $10 \mathrm{~Hz}$ in case we are interested in the R-peaks for instantaneous heart rate computation [25].

\section{B. Choice of Window Length for the Parameters Estimation}

The parameters $V_{d}, D$, and $K$ are estimated via a block leastsquares approach. The block, or window, is sliding in steps of $0.1 \mathrm{~s}$ to provide a smooth estimate of the parameters. The final estimates of $a(t)$ and $s(t)$ are taken at the center of the window resulting in a latency of half the window length (WL).

The WL should be small enough such that the estimation scheme can track variations of the parameters, e.g., a drift in $V_{d}$ or a change of body position affecting the mean value of $C_{c}$ and therefore $K$. The WL should also be large enough such that it does not track and suppress the variations of the signal of interest, i.e., the ECG represented by $s(t)$ in Fig. 8. The WL should thus be larger than the smallest period in the ECG, that is $2 \mathrm{~s}$ if the frequency band is [0.5 40] Hz and $0.1 \mathrm{~s}$ if the frequency band is reduced to [10 30] Hz for R-peak detection. We chose here a WL of $2 \mathrm{~s}$. The parameters can be tracked if they vary with a period well beyond the $\mathrm{WL}$, e.g., $V_{d}$ can vary up to about $0.1 \mathrm{~Hz}$ for a window of $2 \mathrm{~s}$ and up to about $2 \mathrm{~Hz}$ for a window of $0.1 \mathrm{~s}$. The tradeoff regarding the WL is

$$
\frac{1}{\min \text { freq of } \mathrm{ECG}}<\mathrm{WL}<\frac{0.5}{\max \text { freq of } V_{d}} .
$$

A proper $V_{d}$ estimation and tracking can only be done when there is motion at the body-electrode interface, that is when $C_{c}$ varies at frequencies higher than those of $V_{d}$. In static or slowly varying conditions, both the motion $C_{c}$ and the voltage $V_{d}$ are out of the ECG band and do not impact the biopotential measurement.

\section{Alternative Artifact Reduction Using a Feedback Loop}

The $V_{d}$ value, when estimated in real time, can be subtracted at the input of the amplifier of the capacitive sensor (see Fig. 1). As a result, the voltage across the coupling capacitor is reduced together with the amplitude of $a(t)$. The output signal becomes 


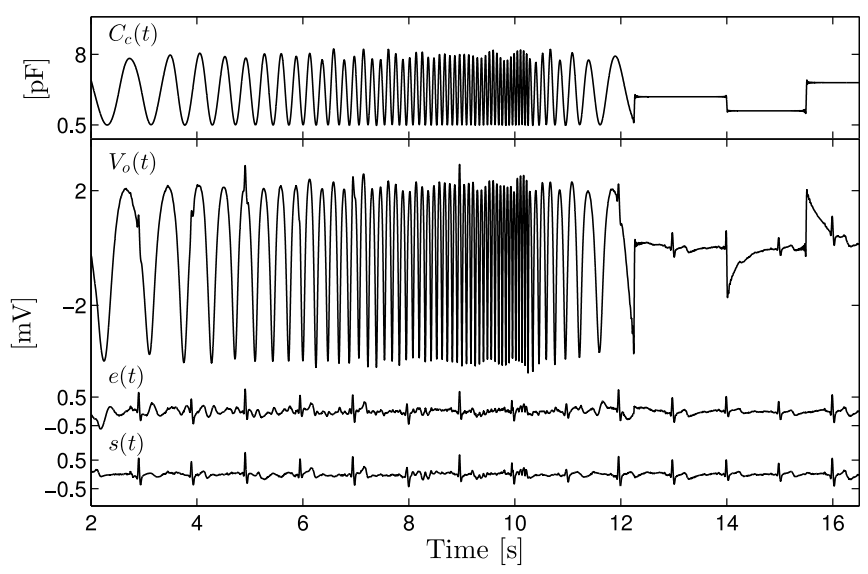

Fig. 9. Example of AR on simulated data. From top to bottom: the variations of the coupling capacitance $C_{c}(t)$; the corrupted signal $V_{o}(t)$ at the output of the capacitive measurement system for $V_{d}=5 \mathrm{mV}$; the processed signal after AR $e(t)$; and the gold standard $s(t)$, which is the output signal in the absence of additive artifact.

$$
\begin{aligned}
V_{\mathrm{o}_{\mathrm{fb}}}(t)=s(t) & +a_{\mathrm{fb}}(t) \text {, where } \\
a_{\mathrm{fb}}(t) & =\frac{V_{d}-\tilde{V}_{d}}{C_{i}+C_{c}(t)} \int_{-\infty}^{t} \frac{d C_{c}}{d u} f(t, u) d u .
\end{aligned}
$$

This idea of using a feedback loop to reduce the dc voltage across $C_{c}$ and the related artifact was proposed earlier in [26]. This method of $V_{d}$ compensation prevents clipping of the system and acts directly at the source of the artifact. In this feedback scheme, the estimate of $V_{d}$ should be taken at the end of the sliding window resulting in a slightly less accurate estimate but no loop latency that would hamper tracking.

\section{RESULTS AND DisCUSSION}

\section{A. Artifact Reduction on Simulated Data}

A chirp motion is chosen to illustrate the performance of our AR method over the whole range of motion frequencies ([0 20] Hz). Some step motion is also simulated to evaluate our method in extreme conditions (first graph of Fig. 9). The output signal $V_{o}(t)$ (second graph of Fig. 9) is so corrupted by the motion artifact that the R-peaks of the ECG are no longer visible. After AR with a 2-s processing window, the ECG signal and its characteristic R-peaks become clearly visible as shown in the third graph of Fig. 9. If our method would work perfectly and thus would entirely remove the additive artifact from $V_{o}(t)$, we would obtain the signal $s(t)$ in the last graph of Fig. 9. This signal only contains distortions due to variations of $C_{c}(t)$ and reducing the signal distortions is not the focus of this paper. The signal $s(t)$ is thus our gold standard. In this specific example, the Pearson's correlation coefficient between the gold standard $s(t)$ and our processed signal $e(t)$ equals 0.72 due to a remaining additive artifact, especially marked for low-frequency motion.

\section{B. Performance Evaluation}

To quantify our method's performance, we compute the signal-to-artifact ratio (S/A) before and after applying our AR method. The difference between these two S/A in $\mathrm{dB}$ determines

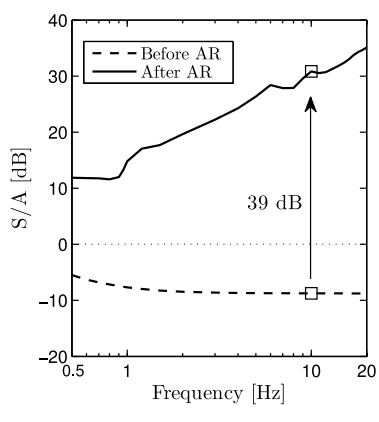

(a)

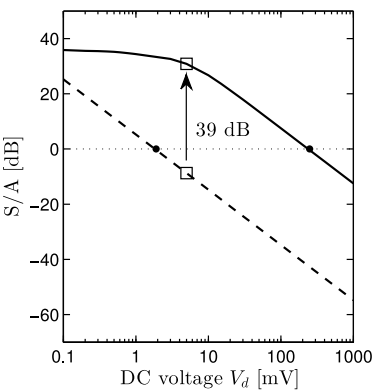

(b)
Fig. 10. AR on simulated data as a function of the frequency of the motion $C_{c}(t)$ and the dc voltage $V_{d}$. The S/A before and after AR is represented by the dashed and solid lines, respectively. In (a), $V_{d}=5 \mathrm{mV}$. In (b), $C_{c}(t)$ varies at $10 \mathrm{~Hz}$.

the amount of AR. The S/A as defined here compares the root mean square (RMS) amplitude of the artifact with the average amplitude of an R-peak in the signal of interest $s(t)$ :

$$
\mathrm{S} / \mathrm{A}=20 \log \left(\frac{\text { mean }(\text { Rpeak amplitude })}{\mathrm{RMS}(\text { artifact amplitude })}\right) .
$$

An S/A lower than $0 \mathrm{~dB}$ means that the mean artifact amplitude is higher than the one of the R-peaks. An S/A higher than $6 \mathrm{~dB}$ is expected to allow a proper R-peak detection since the amplitude of the R-peak will be, in average, twice the one of the artifact. As for the ECG shape, the lowest acceptable S/A depends on the application.

We study our method's performance for different artifact amplitudes and frequency contents. The amplitude of the artifact is directly proportional to $V_{d}$, and the frequency content of the artifact is closely related to the frequency content of $C_{c}(t)$. We thus evaluate our method by making the parameters $V_{d}$ and $C_{c}(t)$ vary between their extreme limit values (as defined in Table I) and computing the S/A before and after AR. Two resulting graphs are shown in Fig. 10. The first one indicates the influence of the motion frequency on our method's performance when $V_{d}$ equals $5 \mathrm{mV}$. The second graph indicates the performance as a function of the artifact amplitude for $C_{c}(t)$ oscillating between 0.5 and $8 \mathrm{pF}$ at a fixed frequency of $10 \mathrm{~Hz}$. In both graphs, the S/A before and after AR is represented by the dashed and solid lines, respectively. An AR up to $47 \mathrm{~dB}$ is possible, depending on the conditions. For $V_{d}=5 \mathrm{mV}$ and $C_{c}(t)$ varying at $10 \mathrm{~Hz}$, our method provides an AR of $39 \mathrm{~dB}$.

Fig. 10(a) shows that the amount of AR increases with the frequency of the artifact. This effect appears to come from the LTI approximation of the dynamic filter: the LTI filter makes a frequency-dependent phase-shift error that decreases with increasing frequency. In this figure, we can also see that the artifact amplitude before AR (dashed line) is attenuated at frequencies below $1 \mathrm{~Hz}$ due to the HP characteristic of the capacitive sensor.

Fig. 10(b) shows that the amount of AR increases with the amplitude of the artifact and becomes constant for high amplitude artifacts. The limitation for small artifact amplitudes is due to the influence of the signal $s(t)$ in the parameters estimation loop. Indeed, for small artifact amplitudes, the amplitude of $s(t)$ 


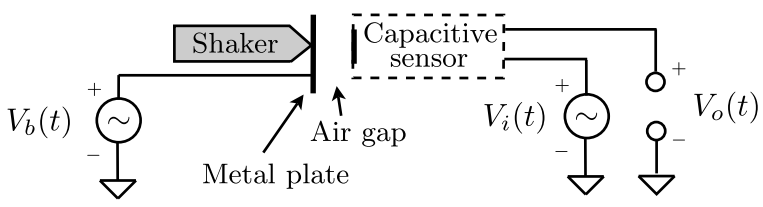

Fig. 11. Lab data acquisition setup. The metal plate and the shaker represent, respectively, the body surface and the body motion. The metal plate is coupled to the capacitive sensor via an air gap. A known 1-kHz signal $V_{i}(t)$, injected from the sensor ground terminal, is used to track the distance variations between the metal plate and the capacitive sensor.

becomes significant, and since $s(t)$ is not a white signal, the estimate $\tilde{a}(t)$ of the artifact $a(t)$ in Fig. 8 is not optimal, leading to a remaining additive artifact in $e(t)$. As for the constant AR, it implies an estimation error that increases linearly with the artifact amplitude. This effect comes from the phase-shift error of the LTI filter that results in a shift between $a(t)$ and $\tilde{a}(t)$ and thus in an estimation error $a(t)-\tilde{a}(t)$ that is proportional to the amplitude of $a(t)$. A $10-\mathrm{Hz}$ motion was chosen as study case because it represents the lowest, hence the most difficult, frequency to be removed when the goal is R-peak detection only. It appears from Fig. 10(b) that a proper R-peak detection $(\mathrm{S} / \mathrm{A}>6 \mathrm{~dB})$ is possible with initial artifacts having an S/A of $-35 \mathrm{~dB}$ or more before $\mathrm{AR}$, i.e., artifacts' amplitudes up to 56 times bigger than the R-peak amplitude.

\section{C. $V_{d}$ Estimate}

The $V_{d}$ estimate is a by-product of our AR scheme. It is estimated automatically during the artifact estimation process. The accuracy of the $V_{d}$ estimate is regarded here for its potential use in a feedback loop as described in Section VIII-C or in a reconstruction scheme as in [13]. The $V_{d}$ estimate accuracy has been observed to increase with the frequency of the artifact. In the extreme case when there is no motion, i.e., at $0 \mathrm{~Hz}$, there is no additive artifact, and $V_{d}$ cannot be estimated as discussed in Section VIII-B. This suggests that an accurate and continuous $V_{d}$ estimation, and thus compensation in a feedback loop, would be optimal in the presence of high-frequency vibrations at the body-electrode interface. A vibrating probe could be used to provide a continuous and subtle high-frequency variation of $C_{c}(t)$ out of the ECG band [26].

\section{Artifact Reduction on Lab Data}

A lab experiment is set up as described in Fig. 11 to validate our AR method experimentally. A periodic artificial ECG signal $V_{b}(t)$ is applied on a metal plate. This plate and the electrode of the capacitive sensor are separated by an air gap and form the coupling capacitor $C_{c}$. Variations of $C_{c}(t)$ are generated by a shaker modulating the distance between the metal plate and the sensor. The values of the system parameters $\left(R_{i}, C_{i}, C_{c}\right.$, and $V_{d}$ ) are unknown. The output signal $V_{o}$ is sampled at $8 \mathrm{kHz}$ and filtered with a 40-Hz low-pass Butterworth filter.

To compare our lab results with our simulation results, a chirp motion covering the full ECG frequency range is generated by the shaker. Since the exact capacitance $C_{c}(t)$ is unknown, the

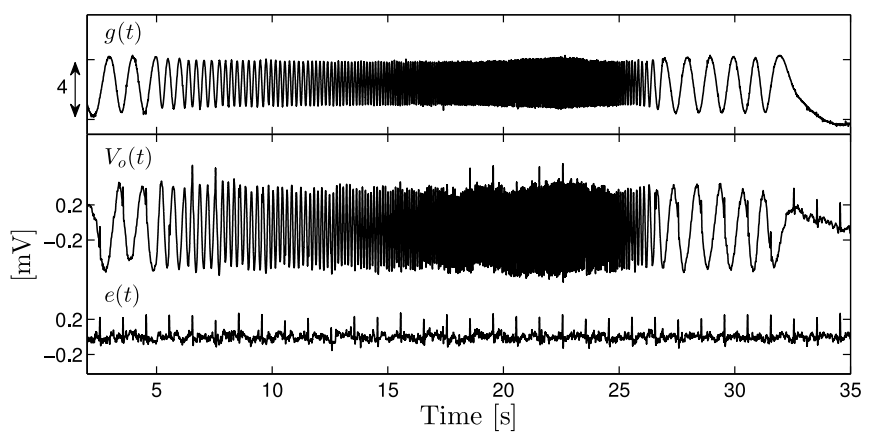

Fig. 12. Example of AR on lab data for a chirp motion. The R-peaks of the ECG signal are masked by the artifact in $V_{o}(t)$. After AR with a 2-s window, the R-peaks become detectable in $e(t)$.

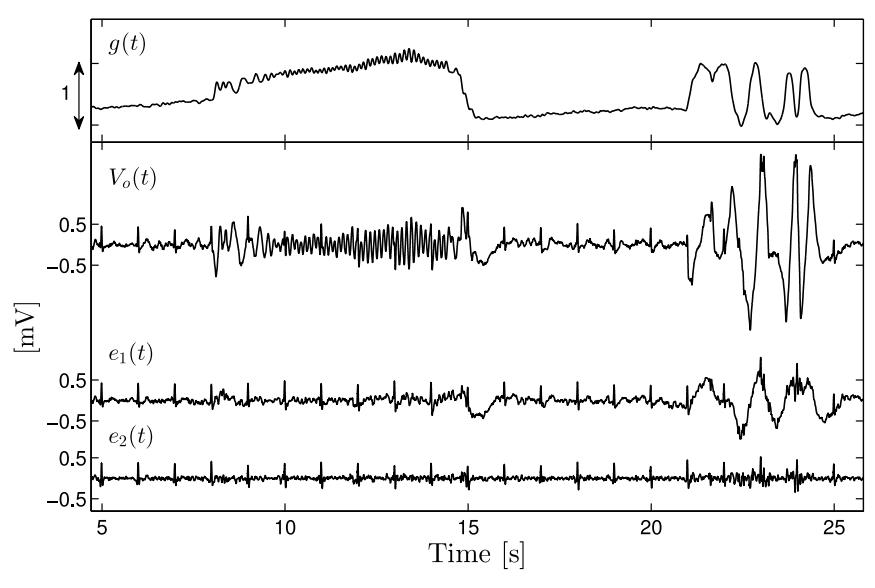

Fig. 13. Example of AR on lab data for a random motion. A 2-s processing window gives $e_{1}(t)$ and a 0.2 -s window gives $e_{2}(t)$.

signal $g(t)$, which is the amplitude modulation of the injection signal as defined in (7), is chosen to illustrate the amount of capacitance changes in Fig. 12. The resulting signals before and after $\mathrm{AR}, V_{o}(t)$ and $e(t)$, are also shown in Fig. 12. The artifact amplitude is in average reduced by $29 \mathrm{~dB}$, which is consistent with our simulation results in Fig. 10. This degree of suppression can also be directly compared to our results obtained in [22] where a reduction of $20 \mathrm{~dB}$ was achieved on the same dataset.

Fig. 13 shows the AR performance for a random motion. The capacitance changes at the body-electrode interface, whose amount is represented by $g(t)$, lead to the corrupted output signal $V_{o}(t)$. From this signal, processing windows of 2 and $0.2 \mathrm{~s}$ are used to obtain $e_{1}(t)$ and $e_{2}(t)$. In static conditions, at seconds 5 and 18 , the shape of the ECG is not altered in $e_{1}(t)$, as guaranteed by the 2-s WL discussed in Section VIII-B. At seconds 10 to 15, the high-frequency artifact is largely reduced, and the R-peaks become detectable again in both $e_{1}(t)$ and $e_{2}(t)$. At seconds 23 to 26, the low-frequency artifacts were reduced by some $9 \mathrm{~dB}$ in $e_{1}(t)$ but not entirely removed. In $e_{2}(t)$, we observe the HP effect obtained when choosing a short processing window of $0.2 \mathrm{~s}$. 


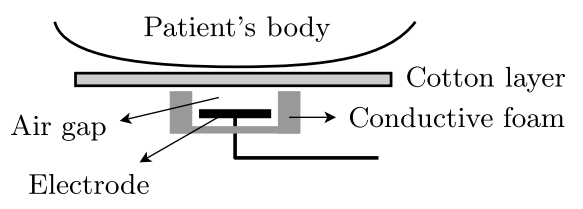

Fig. 14. Real-life data acquisition setup. The electrode is capacitively coupled to the body via the combination of an air gap and a cotton layer. The conductive foam is connected to the system active guard.

\section{E. Artifact Reduction on Real-Life Data}

The method is also tested in real-life conditions. A unipolar ECG is measured on the chest of a male adult. The right wrist is connected to the system ground via a $100-\mathrm{k} \Omega$ resistance for safety. A voltage $V_{i}(t)$ of $1 \mathrm{~V}$ peak-to-peak and $1 \mathrm{kHz}$ is applied on the sensor side. In this way, the maximum current that could flow through the patient's body in case of a malfunctioning sensor is limited to a safe $10 \mu \mathrm{A}$. The choice for the amplitude of $V_{i}(t)$ results from a tradeoff between the patient safety and a high signal-to-noise ratio in $g(t)$. The patient is wearing a tight-fitting cotton T-shirt of about 1-mm thickness. The sensor is positioned under the left nipple and maintained against the chest by an elastic strap. A ring of conductive foam, connected to the sensor active guard, surrounds the sensor and makes contact with the body via the cotton layer as illustrated in Fig. 14. The motion artifact is created by manually and repeatedly pressing the sensor against the patient's body.

An example of a resulting recording is shown in Fig. 15. The R-peaks of the ECG can be seen in $V_{o}(t)$ at seconds 2 to 8 when there is no motion. Our method provides an estimate $\tilde{V}_{d}$ for the dc voltage only when there are sufficient changes of the coupling capacitance, i.e., $\tilde{V}_{d}$ is not given when there is no artifact, as shown in the bottom panel of Fig. 15. Consequently, $\tilde{V}_{d}$ can be used directly to identify the signal segments corrupted by the motion artifact. After processing $V_{o}(t)$ with a 2-s window, the artifact is reduced in $e(t)$ by some $18 \mathrm{~dB}$ at second 11 but a transient remains at second 8.5. In average, the RMS amplitude of the artifact is reduced by $9 \mathrm{~dB}$. Despite a strong amplitude reduction, the artifact is not entirely removed, and the R-peaks do not become clearly visible.

A first reason for the remaining artifact is that the proposed method is based on a simplified model of the system and has its own limits, especially for low-frequency and low-amplitude artifacts as discussed previously in Section IX-B. A second reason is that, due to the thermal noise present in the measurement system, the signal-to-noise-ratio of $g(t)$ is limited (tiny amplitude variations of $C_{c}(t)$ cannot be tracked accurately), which leads to a noisy artifact estimate. Finally, it is highly probable that the artifact in this real-life recording originates, in part, from the other two main sources of artifact which are mentioned in Section II-B and which are outside the scope of this paper. Indeed, the remaining artifact could be partially due to tiny currents (fast variations of $V_{d}$ ) created by the triboelectric effect at the skin-cotton interface. Also, the remaining artifact, and particularly the transient observed at second 8.5 , can be due to the electromagnetic interference created by the motion of the hand (needed to repeatedly apply pressure on the sensor) in the vicinity of the sensor.

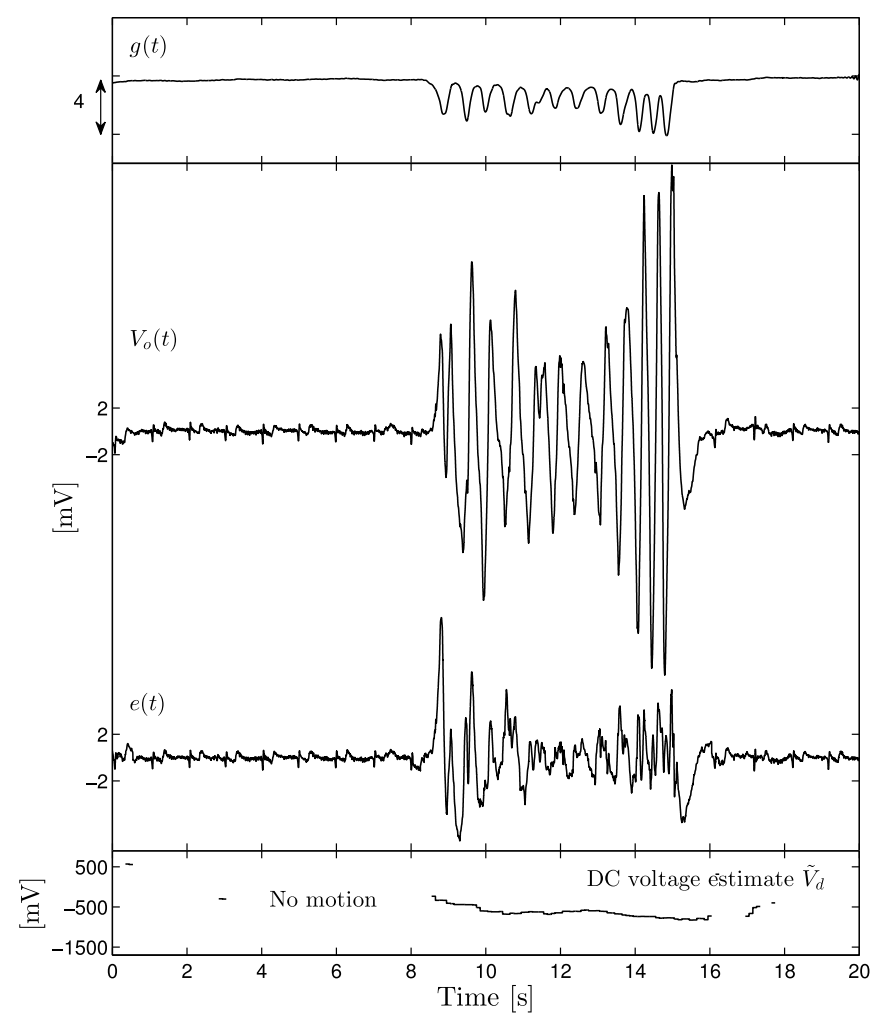

Fig. 15. Example of AR on the ECG recorded from a human chest. $g(t)$ indicates the amount of capacitance changes at the body-electrode interface. $V_{o}(t)$ is the recorded ECG. After processing with a 2-s window, a cleaner ECG $e(t)$ and a dc voltage estimate $\tilde{V}_{d}$ are obtained.

\section{CONCLUSION AND Future Work}

We developed a method to reduce motion artifacts generated by the combination of body-electrode capacitance variations and a dc voltage across the body-electrode interface. The method is based on an injection signal, a model of the system, and an identification scheme.

When the body-electrode interface is an air gap, any variation of the charged coupling capacitance generates two problems simultaneously: a distortion of the measured signal and an additive artifact. The additive artifact tends to be very large and must be dealt with, while the distortion tends to be modest and will not hamper R-peak detection. We showed that an injection signal can be used as a basis to estimate the additive artifact and that a strong AR can be achieved in simulation as well as in a lab setup.

Although the method was designed for an air gap as bodyelectrode interface, we showed that our method also works to a certain extent in real-life conditions with a cotton layer as body-electrode interface. In these conditions, static charges are generated during motion due to rubbing of the fabric against the skin (triboelectric effect). In cases where the artifact under study (microphonics) dominates the triboelectric artifact, as expected in applications where the sensors are embedded in a seat or medical device [4] and more or less pressed against the body, our proposed scheme can accurately estimate a significant part of the artifact. In cases where the triboelectric artifact dominates, 
as expected in ambulatory scenarios and for sensors embedded in clothing, $V_{d}$ will change over time at frequencies that might be in the ECG band. When there are sufficiently high-frequent changes of the coupling capacitance, our method allows to track $V_{d}(t)$ and can hence be used for future studies on the origin and effect of triboelectricity in capacitive measurements. The value of array processing techniques for reducing both microphonic and triboelectric artifacts in an array of capacitive sensors will also be part of future studies.

We showed that the artifact under study is proportional to the dc voltage $V_{d}$. Therefore, limiting $V_{d}$, e.g., by a proper choice of material at the body-electrode interface [9], an active driven grid allowing a faster discharge [9] and/or a $V_{d}$ compensation scheme, will always help in reducing motion artifacts. We showed also that our method can be used to localize, automatically and in an online manner, motion artifacts in real-life ECG measurements, which is a very practical step toward more reliable capacitive ECG measurements.

Besides AR and artifact localization, our method provides knowledge of the model parameters such as the variations of $\frac{C_{c}(t)}{C_{i}}$ and the dc voltage $V_{d}$. The combination of our parameter estimation scheme with the inverse system equations of Heuer et al. [13] could be used not only to remove the additive artifact but also to correct for signal distortions, leading to a high-fidelity ECG reconstruction.

In summary, this paper provides a comprehensive study on distortions, microphonics, and the impact of static charges in capacitive ECG measurement, which is a great step toward a better understanding of the origins of motion artifacts in capacitive recordings as well as the challenges and opportunities related to their removal.

\section{ACKNOWLEDGMENT}

The authors would like to thank G. Janssen, N. Lambert, and A. Fazzi for their precious insights.

\section{REFERENCES}

[1] P. Richardson, "The insulated electrode: A pasteless electrocardiographic technique," in Proc. 20th Annu. Conf. Eng. Med. Biol., 1967, vol. 9, p. 15.7.

[2] Y. G. Lim, K. K. Kim, and K. S. Park, "ECG recording on a bed during sleep without direct skin-contact," IEEE Trans. Biomed. Eng., vol. 54, no. 4, pp. 718-725, Apr. 2007.

[3] M. Walter, B. Eilebrecht, T. Wartzek, and S. Leonhardt, "The smart car seat: personalized monitoring of vital signs in automotive applications," Pers. Ubiquitous Comput., vol. 15, no. 7, pp. 707-715, 2011.

[4] M. Weil, M. Oehler, M. Schilling, and L. Maier, "First clinical evaluation of a novel capacitive ECG system in patients with acute myocardial infarction," Clinical Res. Cardiol., vol. 101, pp. 165-174, 2012.

[5] M. Czaplik, B. Eilebrecht, R. Walocha, M. Walter, P. Schauerte, S. Leonhardt, and R. Rossaint, "The reliability and accuracy of a noncontact electrocardiograph system for screening purposes," Anesthesia Analgesia, vol. 114, no. 2, pp. 322-327, 2012.

[6] Y. Chi, T.-P. Jung, and G. Cauwenberghs, "Dry-contact and noncontact biopotential electrodes: Methodological review," IEEE Rev. Biomed. Eng., vol. 3, pp. 106-119, Oct. 2010.
[7] E. Spinelli and M. Haberman, "Insulating electrodes: A review on biopotential front ends for dielectric skin-electrode interfaces," Physiol. Meas., vol. 31, no. 10, p. S183, 2010.

[8] H. Prance, "Sensor developments for electrophysiological monitoring in healthcare," Appl. Biomed. Eng., 2011.

[9] T. Wartzek, T. Lammersen, B. Eilebrecht, M. Walter, and S. Leonhardt, "Triboelectricity in capacitive biopotential measurements," IEEE Trans. Biomed. Eng., vol. 58, no. 5, pp. 1268-1277, May 2011.

[10] J. Webster, "Interference and motion artifact in biopotentials," in Proc. IEEE Region 6th Conf. Rec., May 1977, pp. 53-64.

[11] O. Casas and R. Pallas-Areny, "Electrostatic interference in contactless biopotential measurements," in Proc. 29th Annu. Int. Conf. IEEE Eng. Med. Biol. Soc., 2007, pp. 2655 -2658.

[12] J. Ottenbacher and S. Heuer, "Motion artefacts in capacitively coupled ECG electrodes," in Proc. World Congr. Med. Phys. Biomed. Eng., 2010 vol. 25/4, pp. 1059-1062.

[13] S. Heuer, D. Martinez, S. Fuhrhop, and J. Ottenbacher, "Motion artefact correction for capacitive ECG measurement," in Proc. Biomed. Circuits Syst. Conf., 2009, pp. 113-116.

[14] B. Eilebrecht, T. Wartzek, J. Willkomm, A. Schommartz, M. Walter, and S. Leonhardt, "Motion artifact removal from capacitive ECG measurements by means of adaptive filtering," in Proc. 5th Eur. Conf. Int. Fed. Med. Biol. Eng., 2012, vol. 37, pp. 902-905.

[15] B. Yin, M. Meftah, and T. J. Ikkink, "Compensation of motion artifacts in capacitive measurement of electrophysiological signals," U.S. Patent 20110137200,2011

[16] K. Mueller, B. Blankertz, G. Curio, and M. Schilling, "Sensor system and methods for the capacitive measurement of electromagnetic signals having a biological origin," U.S. Patent 8193821 , 2012.

[17] D. Buxi, S. Kim, N. Van Helleputte, M. Altini, J. Wijsman, R. Yazicioglu, J. Penders, and C. Van Hoof, "Correlation between electrode-tissue impedance and motion artifact in biopotential recordings," IEEE Sens. J., vol. 12, no. 12, pp. 3373 -3383, Dec. 2012.

[18] T. Wartzek, H. Weber, M. Walter, B. Eilebrecht, and S. Leonhardt, "Automatic electrode selection in unobtrusive capacitive ECG measurements," in Proc. IEEE Comput.-Based Med. Syst., 2012, pp. 1-4.

[19] P. Luna-Lozano and R. Pallas-Areny, "Microphonics in biopotential measurements with capacitive electrodes," in Proc. 32th Annu. Int. Conf. IEEE Eng. Med. Biol. Soc., 2010, pp. 3487-3490.

[20] S. Heuer, S. Chiriac, M. Kirst, A. Gharbi, and W. Stork, "Signal quality assessment for capacitive ECG monitoring systems using body-sensorimpedance," in Proc. Int. Conf. Bioinspired Syst. Signal Process., 2011, pp. 454-458

[21] B. Eilebrecht, J. Willkomm, A. Pohl, T. Wartzek, and S. Leonhardt, "Impedance measurement system for determination of capacitive electrode coupling," IEEE Trans. Biomed. Circuits Syst., vol. 7, no. 5, pp. 682-689, Oct. 2013.

[22] A. Serteyn, R. Vullings, M. Meftah, and J. W. M. Bergmans, "Using an injection signal to reduce motion artifacts in capacitive ECG measurements," in Proc. 35th Annu. Int. Conf. IEEE Eng. Med. Biol. Soc., 2013, pp. 4795-4798.

[23] T. Apostol, Calculus, vol. 1: One-Variable Calculus with An Introduction to Linear Algebra. New York, NY, USA: Wiley, 1967.

[24] E. Spinelli, M. Haberman, P. Garca, and F. Guerrero, "A capacitive electrode with fast recovery feature," Physiol. Meas., vol. 33, no. 8, p. 1277, 2012

[25] S. Abboud and D. Sadeh, "Spectral analysis of the fetal electrocardiogram," Comput. Biol. Med., vol. 19, no. 6, pp. 409-415, 1989.

[26] J. Veen, M. Meftah, N. Lambert, B. M. De Boer, B. Feddes, L. Gourmelon, R. Rietman, and S. A. Husen, "Electro-physiological measurement with reduced motion artifacts," U.S. Patent 20120116 198, 2012.

Authors', photographs and biographies not available at the time of publication. 